\title{
RESTRAINED BUCKLING BEHAVIOR OF CORE COMPONENT IN BUCKLING-RESTRAINED BRACES
}

\author{
J. Wu ${ }^{1,2}$, R.J. Liang ${ }^{1}$, C.L. Wang ${ }^{2,3}$ and H.B. Ge $e^{2,3^{*}}$ \\ ${ }^{1}$ Key Laboratory of Concrete and Prestressed Concrete Structures of the Ministry of Education, \\ Southeast University, Sipailou 2, Nanjing 210096, China \\ 2. International Institute for Urban Systems Engineering, Southeast University, Sipailou 2, Nanjing 210096, China \\ 3. Department of Civil Engineering, Meijo University, Tempaku-ku, Nagoya 468-8502, Japan \\ *(Corresponding author: E-mail: gehanbin@meijo-u.ac.jp)
}

Received: 30 May 2011; Revised: 27 July 2011; Accepted: 12 August 2011

\begin{abstract}
In order to investigate the bending moment of the core plate as well as the contact force between the core plate and the restraining component of a Buckling-Restrained Brace member, focus is set to the multi-wave buckling behavior of the core plate under increasing axial load. Based on the equilibrium equation of a segment subjected to axial and lateral forces, equations are derived to describe the deflection curve, the distribution of the moment of the core plate and the contact force. Discussion about these equations reveals the process of multi-wave buckling of the core plate, which is different with that of a free buckling without the lateral restraining component. The core plate experiences point contact, line contact and new wave generation repeatedly, and the axial loads corresponding to different states are given. A calculation example illustrates that the derived equations agree with numerical results, and an experimental result is used to verify the equations.
\end{abstract}

Keywords: Buckling-restrained brace, Restrained buckling, Contact, Multi-wave buckling

\section{INTRODUCTION}

Buckling-Restrained Brace (BRB) is a kind of metal-yield energy-dissipation device. The core plate of a BRB, which sustains the axial tension and compression under strong earthquakes, is expected to yield before the main structural members. Because BRBs dissipate the energy inputted by the earthquake through the post-yield hysteretic behavior, structural seismic responses decrease and main structural members are kept away from severe damage. Due to the advantages of BRBs such as stable hysteretic characteristics, simplicity in installation and low cost, they have been applied extensively in many building structures as well as bridges in Japan, North American and China (Uang et al. [1], Lai and Tsai [2], Xie [3], Luo et al. [4], Chen et al. [5]).

As shown in Figure 1, a BRB mainly consists of the core plate, the unbonding material and the restraining component (Watanabe et al. [6]). The core plate, commonly made of the low yield metal with a rectangle or cross sectional shape, is designed to resist the axial force. The restraining component is designed to enhance the bending stiffness of the BRB and restrict the buckling amplitude of the core to prevent the BRB from overall buckling. Furthermore, the core plate is expected to yield in both compression and tension and exhibits the stable and repeatable elasto-plastic hysteretic behavior. As shown in Figure 1, the restraining component is manufactured with a steel tube filled with concrete or mortar. Between the core plate and the restraining component, there is an unbonding material to reduce the friction and eliminate the force transferred from the core plate to the restraining component when the BRB is subjected to the compressive force. 


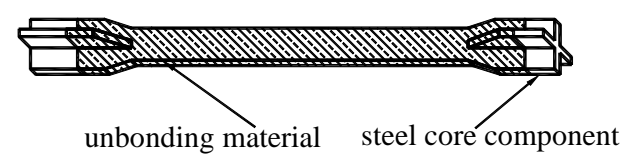

(a)

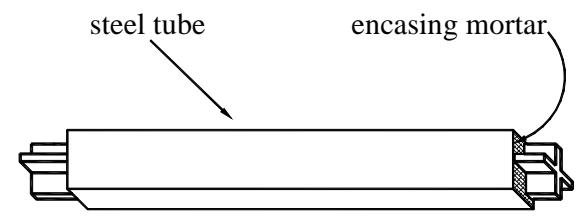

(b)

Figure 1. Concept of a Type of Buckling-Restrained Brace (Watanabe et al. [6])

Due to the confinement of the restraining component, the compressed core plate will generate high-order, slight-amplitude and multi-wave buckling shape, which is a kind of restrained buckling or constrained buckling. The buckling mode shape of a restrained buckling member cannot develop freely like Euler buckling because the existence of the restraining component, which lead to great changes of the behavior of the core plate (Guo and Ren [7]). Sridhara [8] proposed a compressive member embedded in a tube and derived the buckling load when the point contact occurs between the core plate and the tube, using the linear elastic small deflection theory and supposing that the deflection is infinity when the member buckles. Prasad [9] carried out a series of compression test of the typical sleeved member with fixed ends, and obtained the longitudinal force-displacement curve, which demonstrated a temporary drop in load when the core jumped from on mode to other. Domokos et al. [10] analyzed the force-displacement curve of the compressed elastic beam between the rigid, frictionless side-walls through theoretical formula, numerical analysis and tests. Ha [11] studied the rigid constrained buckling of the elastic column in a rigid tube when the point contact and line contact occur, using the calculation model like Domokos's [10]. Chai [12] carried out the theoretical analysis and experimental test on the post-buckling behavior of the compressed elastic column between two straight, rigid and frictionless side-walls in 1998, and obtained the similar force-displacement curve as given in the reference [10]. Shen and Deng [13-14] proposed the mechanical model when the line contact occurs between the core plate and the flexible outer tube.

Generally, a BRB member will break due to the low cycle fatigue of the core plate when subjected to the reversed cyclic loading with the large strain amplitude. It is obvious that the capacity of the low cycle fatigue is related to the maximum strain encountered during the loading procedure, such as the maximum compressive stain of the core plate, which is the combination of the average strain due to the axial force and the bending strain due to the curvature of the multi-wave buckling. The significant contact force and the consequent friction between the core plate and the restraining component take place when the core plate is subjected to the compression force. Consequently, the restraining component sustains part of the axial force. However, the contact and friction forces disappear because the buckled waves of the core plate under the tension are straightened. So, the compressive force of the core plate is greater than its tensile force with the same strain amplitude and the hysteric behavior of the BRB is asymmetric.

The low-cycle fatigue property and the hysteretic behavior are greatly influenced by the behavior of the core plate, indicating that the restrained buckling behavior of the core is an important problem in the researches of the BRB. Although the experiment demonstrated that the multi-wave buckling occurs in the core plate (Usami et al. [15]), the buckling mechanism of the core plate needs to be further investigated. Based on the compressive equilibrium equation of a member restrained by contact, this paper derives the deflection curve of the core plate, which is contacted with two rigid restraining components in point and in line, and reveals the process and mechanism of the multi-wave buckling of the core plate. 


\section{ASSUMPTIONS}

When analyzing the behavior of a BRB member, it should be included in consideration of the geometric nonlinear effect caused by the buckling behavior of the core plate in compression, the contact nonlinear effect caused by contact restraint of the lateral restraining component, and the material nonlinear effect while the BRB develops post-yield strain. Therefore, the mechanical simulation of the BRB's core component is a complex nonlinear problem. On the basis of the actual conditions for a common BRB member, several assumptions are adopted in this paper as follows:

1) The bending deformation of the restraining component is neglected. Generally, the bending stiffness of the restraining component is much greater than that of the core plate. The lateral deformation of the restraining component caused by the contact forces is extremely slight, so that it can be ignored.

2) Two ends of the core plate do not rotate during the loading process. This is due to the large bending stiffness at the ends, which are usually enhanced with stiffeners in the vertical plane.

3) Two ends of the core plate are constantly close or contacted with one side of the restraining component. The original position of the core may be parallel to the restraining component, or a little deviate in the range of the clearance. However, when the core buckles in compression and subsequently contacts with the restraining component, the contact force in the middle makes both ends of the core contact with the opposite side of the restraining component. Obviously, only the symmetric buckling mode shape is considered in this assumption. The reference [10] implied that the duration of an antisymmetric buckling mode was very short so that the wave was unstable and would jump into higher modes randomly. In this paper, only symmetric buckling modes are considered.

4) The elastic modulus of the core plate remains constant. The modulus keeps constant before the core plate yields, and it decreases as the core experiences post-yielding, especially during the severe tension-compression cyclic loading with large inelastic strains. The difficulty of the inelastic buckling is that the modulus in the loading zone of a section may differ from that in the unloading zone, which causes the buckling load difficult to solve. However, according to the Shanley concept, the tangent modulus solution of inelastic buckling load is the lower limit and close to the experimental results. It implies that as inelastic buckling is under consideration, the elastic modulus in corresponding equations can be simply replaced by the tangent modulus to obtain a reasonable solution.

5) The axial deformation of the core in compression is neglected. The length of the compressed core plate will decrease due to the bending caused by buckling and the deformation caused by the axial force. For a BRB member, the clearance between the core plate and the restraining component, which is the maximum amplitude for the buckling deformation, is very small, so that the bending-caused deformation is slight and negligible. The compressed deformation caused by the axial force is also very small and can be ignored.

6) The friction between the core plate and the restraining component is neglected. Due to the lateral deformation of the core plate and consequently contact forces between the core plate and the restraining component, the friction forces arise inevitably as the core plate buckles under compression. However, the magnitude of the friction forces is relatively small compared with the axial force the BRB member sustained, and the existence of the friction will make the problem complex. In order to simplify the derived equations, this study does not consider the friction effects. 


\section{DEFLECTION SHAPE OF CORE COMPONENT DURING BUCKLING PROCESS}

\subsection{Deflection Equation under Axial and Lateral Forces}

According to the assumptions (1) to (3), a BRB in its initial state is sketched in Figure 2(a), where $L, E$ and $I$ are the length, elastic modulus and moment of inertia of the core plate, respectively, and $2 y_{0}$ is the clearance between the core plate and the far-side restraining component. As presented in Figure 2(b), when the increasing axial compression force $P$ reaches to the Euler force $P_{\mathrm{E}}$ of the slender core plate with the $L$ length, it buckles and makes critical contact with the far-side restraining component. The Euler force is given as follows:

$P_{\mathrm{E}}=\frac{4 \pi^{2} E I}{L^{2}}$

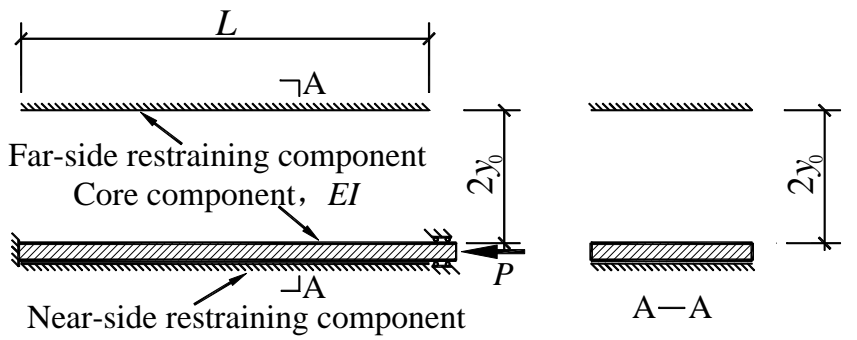

(a)

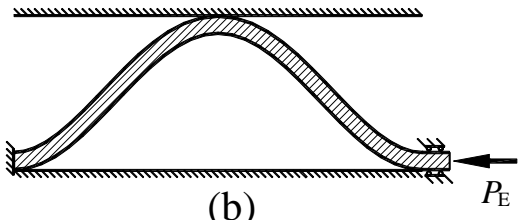

(b)

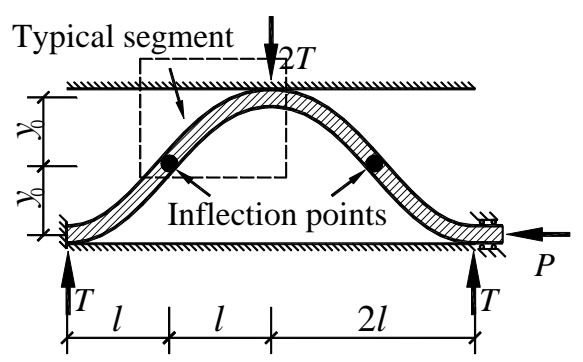

(c)

Figure 2. Buckling Process of Core Component

The deflection of the core plate tends to be developed with the increase of the axial force $P$, but the amplitude of the deformation is suppressed by the lateral restraining component. Therefore, the contact force generates between the core plate and the restraining component. As given in Figure 2(c), $T$ is the shear force in the free segment between two contact points, and the distance between the two adjacent contact points is defined as $2 l$. Considering the symmetry, any segment between the inflection point and the contact point is identical, and one is illustrated in Figure 3. Obviously, the segment under the combination of the axial force $P$ and the contact force $T$ satisfies: 


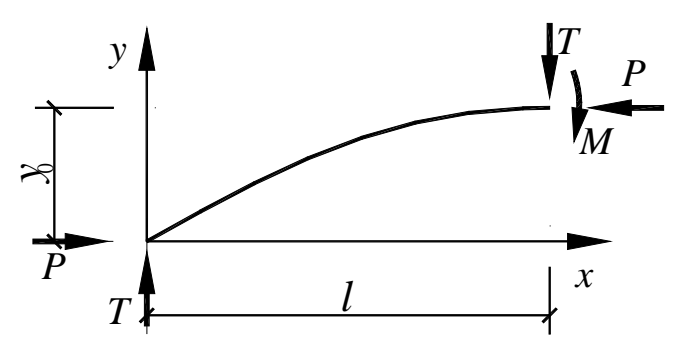

Figure 3. Calculating Diagram of Segment under Axial and Contact Forces

$$
P y-T x+E I \frac{d^{2} y}{d x^{2}}=0
$$

According to the boundary conditions $(x=0, y=0)$ and $\left(x=l, y=y_{0}\right)$, the deflection equation of this member can be obtained as:

$$
y=\frac{\sin \left(\alpha \frac{x}{l}\right)\left(P y_{0}-T l\right)}{P \sin \alpha}+\frac{T}{P} x
$$

where $\alpha$ is the dimensionless nominal axial force relative to the Euler buckling load:

$$
\alpha=\frac{\pi}{2} \sqrt{\frac{P}{P_{\mathrm{E}}}}
$$

Specially, in the state of critical point contact, there are $T=0$ and $\alpha=\pi / 2$, which lead to the deflection equation of this situation as:

$$
y=y_{0} \sin \left(\frac{\pi}{2} \frac{x}{l}\right)
$$

\subsection{The Point Contact Situation}

According to the symmetry of the core plate about the middle contact point, there is:

$$
\left.y^{\prime}\right|_{x=l}=0
$$

Substituting condition (6) into Eq. 3 gives half of the contact force in the middle contact point,

$$
T=\frac{P y_{0}}{\alpha-\tan (\alpha)} \frac{\alpha}{l}
$$

The deflection curve of the segment can be derived by substituting Eq. 7 into Eq. 3. That is, 
$y=y_{0} \frac{1}{\tan \alpha-\alpha}\left(\frac{\sin \left(\alpha \frac{x}{l}\right)}{\cos \alpha}-\alpha \frac{x}{l}\right)$

At the same time, the moment of the segment under this condition can be derived as follows:

$$
M=P y-T x=-E I \frac{d^{2} y}{d x^{2}}=P y_{0} \frac{1}{\sin (\alpha)-\alpha \cos (\alpha)} \sin \left(\alpha \frac{x}{l}\right)
$$

Figures 4 and 5 compare the deflection and moment curves of the core segment when $\alpha / \pi$ is 0.5 , 0.6, 0.7, 0.8, 0.9 and 1.0, respectively. As shown in these figures, the core segment and the restraining component always keep the state of the point contact in the above loading range. The increase of the axial force changes the deflection shape gradually, and makes the deflection curve plump and close to the restraining component around the contact point. Moreover, the position of the maximum moment moves from the contact point to the midpoint of this segment. When $\alpha=\pi$ and $P=4 P_{\mathrm{E}}$, the moment or the curvature of the core segment at the contact point decreases to 0 , indicating that the condition $\alpha=\pi$ is the end of the point contact state as well as the beginning of the line contact state, as given in the reference [13]. In other words, the point contact between the core plate and the restraining component occurs when $0.5 \pi<\alpha<\pi$.

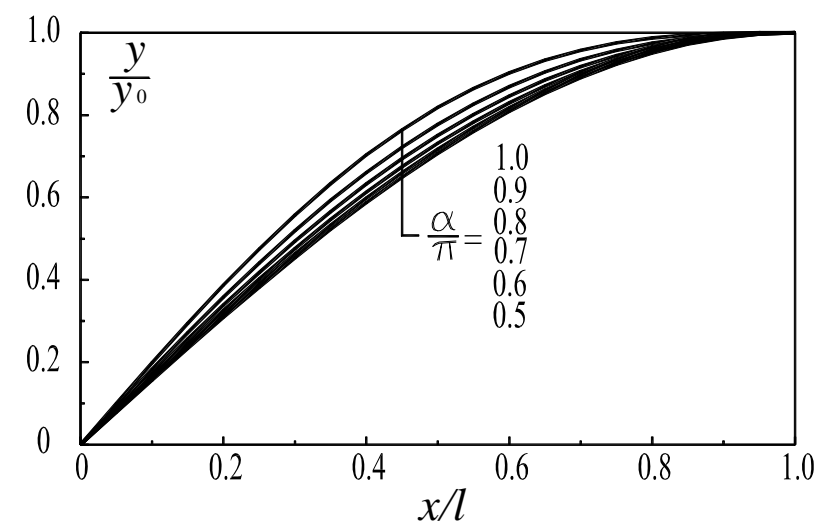

Figure 4. Deflection Curves under Different Axial Forces

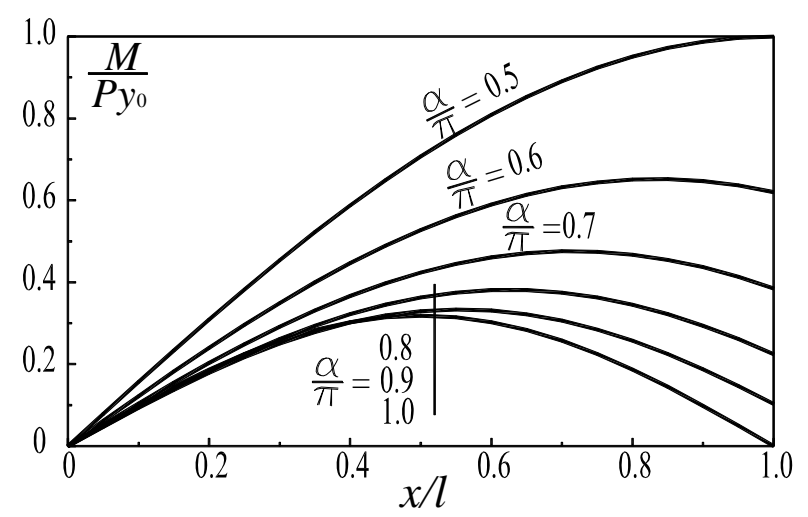

Figure 5. Moment Curves under Different Axial Forces 
It is obvious that the difference between the restrained buckling and the free buckling can be investigated in the above derivation. As $P=4 P_{\mathrm{E}}$, the segment buckles at the second order buckling mode for the free buckling, where the deflection curve performs two complete waves; while in the restrained buckling, there is only one complete wave under this load, because the contact force, which can be regarded as an intermediate action of the slender segment, enhances the lateral supporting stiffness to the core segment.

\subsection{State of Line Contact}

After the situation of $\alpha=\pi$, the increase of the axial force $P$ tends to curve the segment plumper, resulting in the line contact between the segment and the restraining component. The free length of the segment that does not contact with the restraint, $2 l$, tends to be reduced, and the corresponding Euler buckling load of the free segment is:

$$
P_{\mathrm{E}}^{\prime}=\frac{\pi^{2} E I}{4 l^{2}}
$$

Note that the free length in this situation is smaller than that in the point contact state. At this situation, only two concentrated contact forces exist at the two ends of contacted line, while in the rest part of the line there is no distributed contact force because the deflection curve is flat. The deflection equation of the free segment is the same as that in the end of point contact state except for the difference in the length of free segment. Owning to $\alpha=\pi$, the deflection equation at this point is:

$$
y=y_{0}\left[\frac{1}{\pi} \sin \left(\pi \frac{x}{l}\right)+\frac{x}{l}\right]
$$

In the situation of line contact, when investigating the nominal axial force $\alpha$ with Eq. 4 , the $P_{\mathrm{E}}$ should be replaced with $P_{\mathrm{E}}^{\prime}$, that is,

$$
\alpha=\frac{\pi}{2} \sqrt{\frac{P}{P_{\mathrm{E}}^{\prime}}}
$$

According to that $\alpha=\pi$, the half of the free length, $l$ can be obtained by combining the Eq. 10 and Eq. 12, that is,

$$
l=\pi \sqrt{\frac{E I}{P}}
$$

The deflection curve of the core plate in this situation is shown in Figure 6.

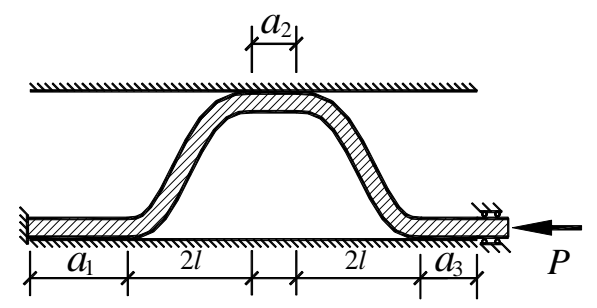

Figure 6. Illustration of State of Line Contact 


\subsection{Generation of New Wave}

In Figure 6, $a_{1}, a_{2}$ and $a_{3}$ are the lengths of the line contact segments. Note that the sum of the contacted lengths may be distributed arbitrary in these three segments. That is to mean, in some cases, $a_{1}$ may be the largest one among the three, while in other cases that is $a_{2}$ or $a_{3}$. The reason of this phenomenon lies in the total strain energy stored in the core plate. Given the same total length and shape of the free segments, the strain energy remains constant in spite of how the segments are distributed in longitude. As the axial force increases, the contact lines extend while each free part behaves like an independent segment with fixed ends without intermediate actions. If the contacted line extends sufficiently to occur a new buckling, the new waveform will be generated.

Obviously, in the event that all of the contacted line concentrates on one segment, this segment holds the maximum length to generate a potential buckling. Without loss of generality, all contact lengths are assumed to be concentrated to $a_{1}$, while $a_{2}=a_{3}=0$, as shown in Figure 7(a). According to the above discussion, at the critical state of the new buckling, the length of the contacted line is controlled as follow:

$P=\frac{4 \pi^{2} E I}{a_{1}^{2}}$

While according to the 5th assumption, the total length of the core plate remains constant, that is

$a_{1}=L-4 l=L-4 \pi \sqrt{\frac{E I}{P}}$

Substituting Eq. 15 into Eq. 14, while the second wave generates, the axial force is:

$P=36 \frac{\pi^{2} E I}{L^{2}}=9 P_{\mathrm{E}}$

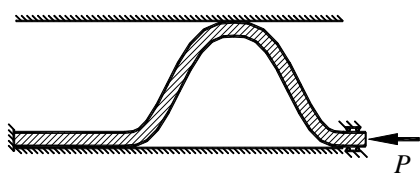

(a)

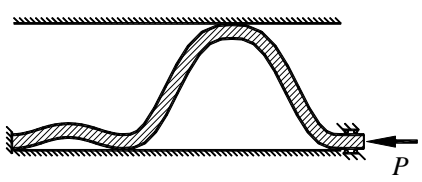

(b)

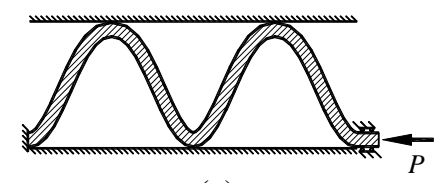

(c)

Figure 7. Process of New Wave Generation

Substituting Eq. 16 into Eq. 13 and Eq. 15, respectively, half the length of the free segment and the length of contact line can be derived as follows: 
$l=\frac{1}{6} L, \quad a_{1}=\frac{1}{3} L$

According to Eq. 17, the deflection curve of the core plate behaves asymmetric at the moment of the new wave generating. One part of the core plate is in the point contact situation with contact forces at the ends, while the other part is in the neutral equilibrium state of the beginning of a new buckling, as shown in Figure 7(b). However, this status is not stable because of the high potential energy, resulting in a quick alteration to a symmetrical contact state with two waves, which occupies lower energy, as shown in Figure $7(\mathrm{c})$. In this situation, there are $l=L / 8$ and $P=36 \pi^{2} E I / L^{2}$. Substituting these parameters into Eq. 12 gives the nominal axial load of this state, that is,

$\alpha=\frac{3}{4} \pi$

As the above nominal axial force satisfies the condition $0.5 \pi<\alpha<\pi$, it indicates that the core plate switches to a point contact state as soon as the new wave occurs. After that, an increase of the axial force will drive the core behaving as two separate segments, each of which is as that described in Figure 2(a), and with the length of $L / 2$. At the same time, the nominal axial force $\alpha$ increases to $\pi$, then the line contact will occur and a new wave will be generated subsequently.

\subsection{Multi-wave Buckling}

As discussed above, the point contact, line contact and new wave generation occur repeatedly under the increasing axial compression force for the restrained core component. If $n$ waves have been generated for the core, each wave will develop as a separate segment with the length of $L / n$. According to the condition $\alpha=\pi$, the axial force at the end moment of point contact situation is:

$P=4 P_{\mathrm{E}}^{\prime}=(2 n)^{2} P_{\mathrm{E}}$

Similarly, at the moment of generating a new wave, considering the length consistent condition of the core plate, that is, the summation of the contacted length and the waved-segment lengths equals to the original length, there is,

$a_{1}=L-4 n \pi \sqrt{\frac{E I}{P}}$

Substituting Eq. 20 into Eq. 14, gives the axial force as the $n+1$ th wave generates

$P=(2 n+1)^{2} P_{\mathrm{E}}$

In the process of the axial force increasing, the value of the dimensionless nominal axial force relative to the free length of the current wave, $\alpha$, always keeps between $\pi / 2$ and $\pi$. The relationship between $\alpha$ and the actual axial forces is shown in Figure 8. In this figure, the curved lines, which are very close to slash lines in Figure 8, represent the slow alterations of the deflection shapes in their current waveforms, while the horizontal lines represent the gradual increases of the contacted lengths, and the vertical lines represent the sudden increases of the number of waves. As a result, a point contact between the core plate and the restraining component occurs and continues when the axial force increases from $(2 n-1)^{2} P_{\mathrm{E}}$ to $(2 n)^{2} P_{\mathrm{E}}$, and the line contact between them 
occurs and continues when the axial force increases from $(2 n)^{2} P_{\mathrm{E}}$ to $(2 n+1)^{2} P_{\mathrm{E}}$.

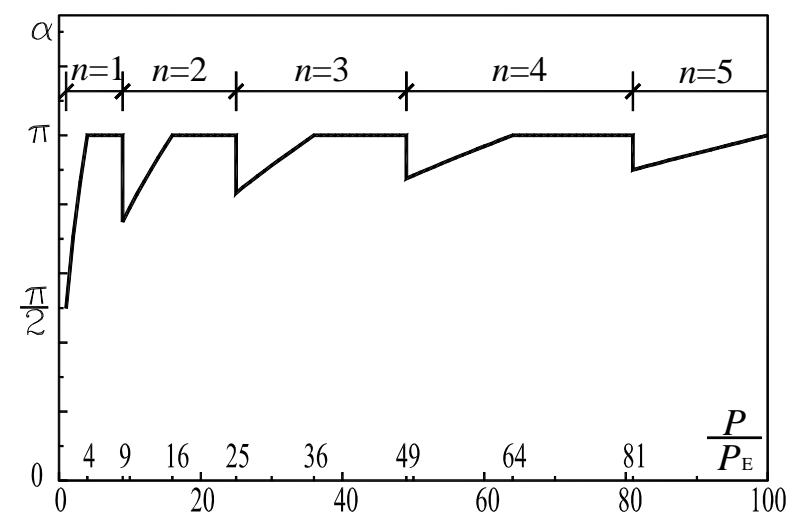

Figure 8. Relationship between $\alpha$ and Axial Force

\subsection{Contact Force}

As illustrated in Figure 2(c), the contact force resisted by the core plate depends on the shear force of the curved segment, $T$. In case of the point contact between the core plate and the restraining component, the core plate can be regarded as $n$ identical waves, each of which with the length of $L / n$, thus

$l=\frac{L}{4 n}$

Applying Eq. 22 in Eq. 7, the shear force of the free segment is:

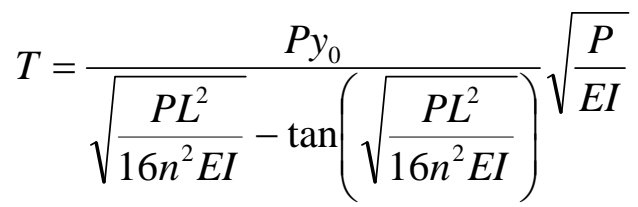

And in case of line contact, applying $\alpha=\pi$ and $l=\pi \sqrt{\frac{E I}{P}}$ in Eq. 7, the shear force of the free segment can be derived as,

$T=\frac{P y_{0}}{\pi} \sqrt{\frac{P}{E I}}$

Several conclusions can be drawn by investigating the Eq. 23 and Eq. 24. The contact force is proportional to the clearance between the core plate and the restraining component. On the other hand, the contact force is monotonic increasing about the axial force applied to the core plate, and monotonic decreasing about the bending stiffness of the core plate. That is, the contact force will increase as the clearance increases, or as the axial force increases, or on the other hand as the bending stiffness of the core plate decreases. These findings are meaningful to design a BRB member, because the contact force is the main reason that causes the friction between the core plate and the restraining, while the friction, which causing the restraining component to resisting the axial force, leads to larger compressive yield capacity than predicted. For example, in order to minish the friction, a reasonable solution can be obtained by detailing the clearance as small as possible, or strengthening the bending stiffness of the core plate. 


\section{NUMERICAL EXAMPLE}

A core plate, with the width and the thickness of $0.1 \mathrm{~m}$ and $0.01 \mathrm{~m}$ respectively, is encased between two rigid, frictionless restraining components, with the clearance of $0.02 \mathrm{~m}$. The original location of the core plate satisfies the $3^{\text {rd }}$ assumption mentioned above. The elastic modulus of the material is $E=2 \times 10^{5} \mathrm{~N} / \mathrm{mm}^{2}$, and the Euler buckling load is $P_{\mathrm{E}}=65.797 \mathrm{kN}$.

Figure 9 illustrates the maximum moment of the core plate calculated by Eq. 9 with the increasing of the axial force. The shear forces of the free segment calculated by Eqs. 23 or 24 are presented in Figure 10.

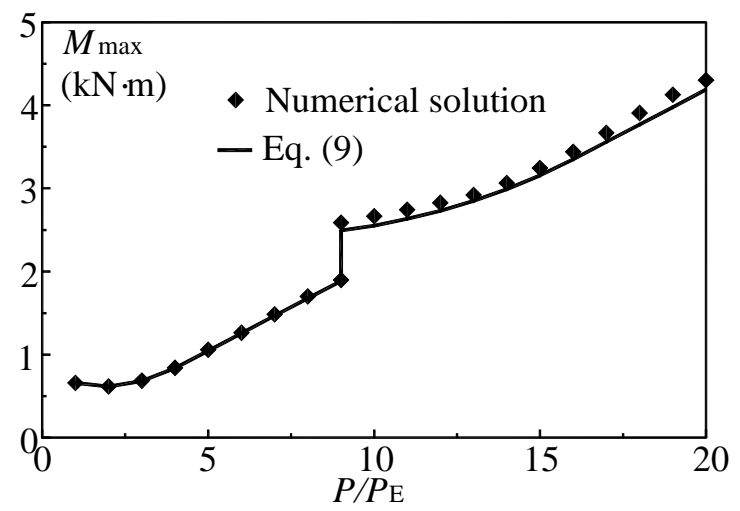

Figure 9. Relationship between Maximum Moment of Core and Axial Force

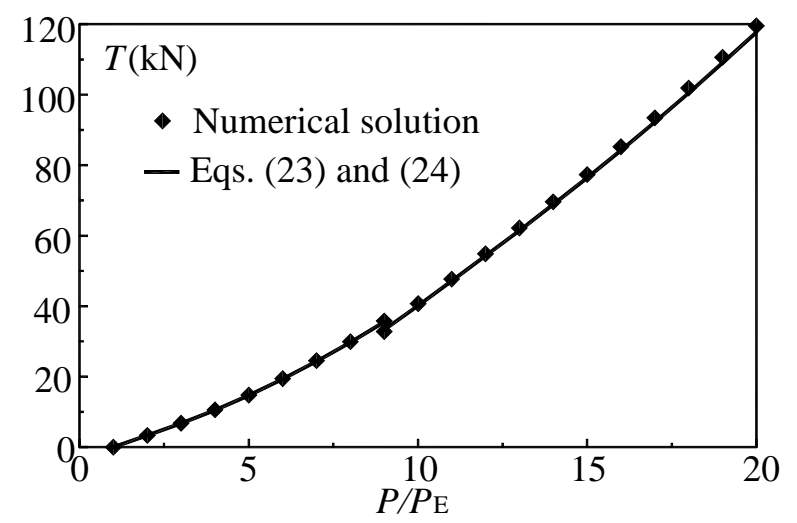

Figure 10. Relationship between Contact Force and Axial Force

As shown in Figures 9 and 10, an abrupt shift occurs both to the maximum moment and the contact force at $P / P_{\mathrm{E}}=9$. This phenomenon is caused by the mode shape jumping of the core plate from one to two waves at this load level.

A corresponding numerical model is established using the program ANSYS. The core plate and the rigid restraining components are modeled using the Beam189 element. They are divided into 60 elements along the longitude and covered with Conta176 and Targe170 elements, respectively. One end of the core is fixed, and the other end is clamped and subjected to an increasing axial force. The maximum moments of the core and the shear forces of the free segment under different axial force levels are also plotted in Figures 9 and 10, respectively, describing the consistency with the theoretical results. From the figures, the maximum error between the maximum moments calculated by the proposed equations and the numerical results is $4.3 \%$, while that of the shear force is $1.4 \%$. These disparities may be due to the error introduced in the nonlinear iteration of the numerical simulation, as well as the shortening of the core plate in longitude, which is neglected in this paper. 


\section{EXPERIMENTAL VERIFICATION}

A sandwich BRB specimen is experimentally studied to verify the theoretical derivation. The core component of the specimen is a steel plate with the thickness of $10 \mathrm{~mm}$, the width of $100 \mathrm{~mm}$, and the length of the yielding segment $L$ of $1075 \mathrm{~mm}$. The measured elastic modulus of the steel is $2.04 \times 10^{5} \mathrm{~N} / \mathrm{mm}^{2}$. The restraining component is composed of two cover plates with the thicknesses of $14 \mathrm{~mm}$ connected by high-strength blots through two side plates with the thicknesses of $12 \mathrm{~mm}$. The core plate is covered with 1-mm-thick butyl rubber tapes as the unbonding material to reduce the friction force between the core and restraining components. The schematic diagram of this specimen is shown in Figure 11.

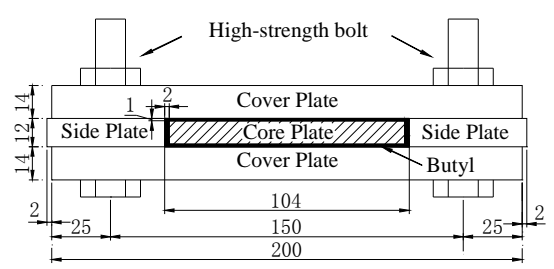

(a) Cross Section
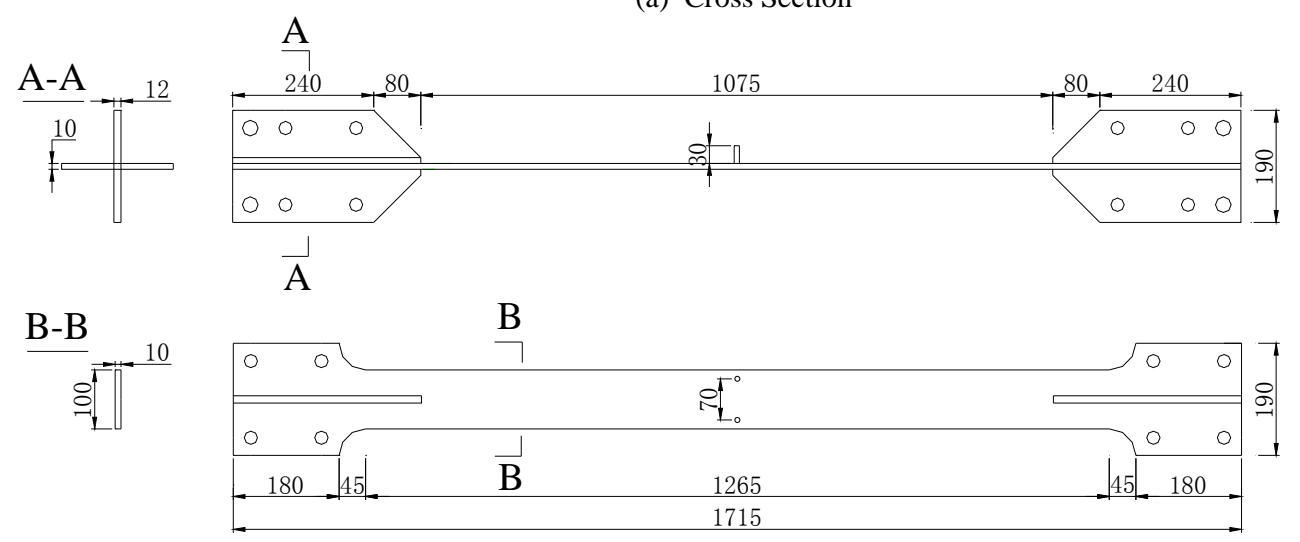

(b) Core Plate

Unit: mm

Figure 11. Schematic Diagram of BRB Specimen

When the core plate is under the compressive loading, the multi-wave buckling occurs. The unbonding material is pressed upon the restraining components and the final buckling shape is printed on the butyl rubber tape. A low-cycle static loading test with the strain amplitude of $2 \%$ is conducted, and the hysteretic curve is plotted in Figure 12. As shown in this curve, the tangent modulus after yielding, $E_{t}^{\prime}$, can be measured as $3.34 \times 10^{3} \mathrm{~N} / \mathrm{mm}^{2}$, and the maximum compressive loading $P$ is $393 \mathrm{KN}$. According to the $4^{\text {th }}$ assumption, the tangent modulus, $E_{t}^{\prime}$, is used in Eq. 1 as $E$, so that $P_{E}$ is calculated as $0.95 \mathrm{KN}$ and $P / P_{E}$ is equal to 413 , which is between $(2 \times 10)^{2}$ and $(2 \times 10+1)^{2}$. It indicates that the number of the final buckling wave is 10 .

After the test, the unbonding material attached on the surface of the restraining component can reflect the multi-wave buckling shape clearly. As presented in Figure 13, the number of the final buckling wave is 10 . It demonstrates that the theoretical result is identical with the test one. 


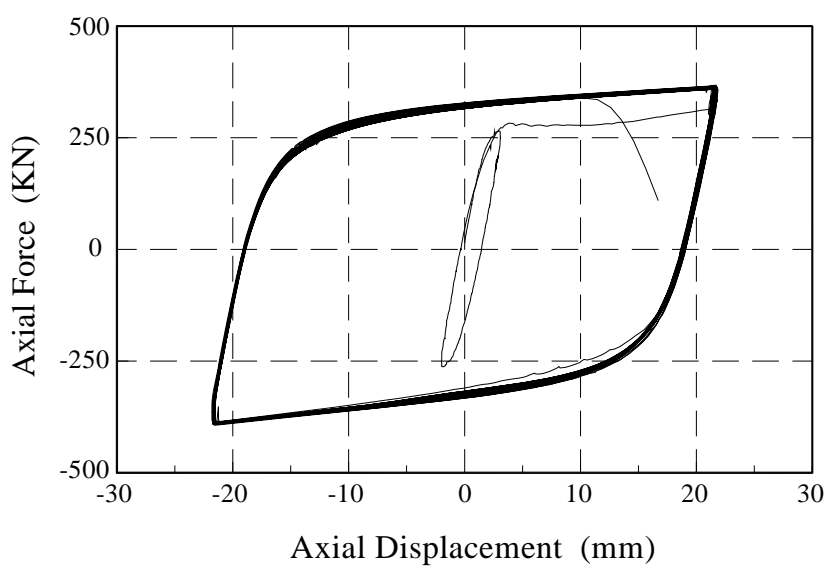

Figure 12. Hysteretic Curve of the BRB

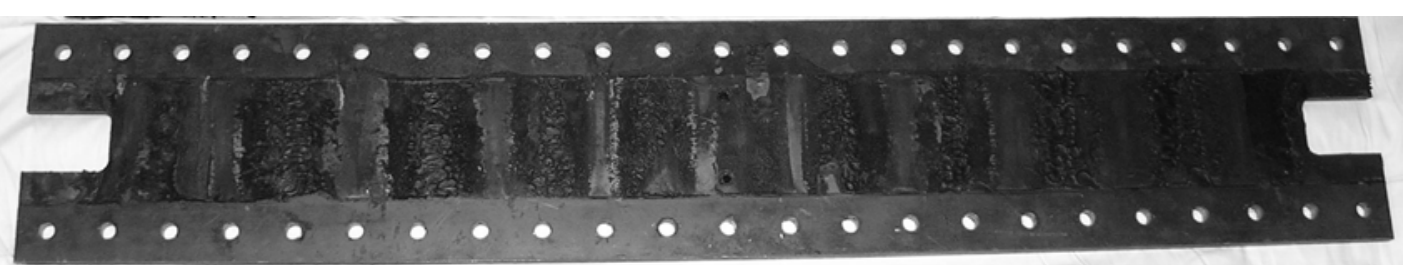

Figure 13. The Buckling Wave Print at the Cover Plate

\section{CONCLUSIONS}

Based on mechanical characteristics of the BRB member, some reasonable assumptions are introduced to establish the equilibrium equation of a free segment of the core under the combination of the axial compression and lateral contact forces. The equations used to describe the deflection curve, the moment distribution and the contact force of the core in the state of the point contact and the line contact, are derived. The discussion about the derived equations reveals the process and mechanism of the multi-wave buckling behavior under the increasing axial force. Some conclusions are summarized as follows:

1) The feature of a restrained buckling is different with that of a free buckling because the contact force acts like an intermediate support of the slender member, which strengthens the lateral stiffness of the member.

2) As the axial force increasing, the point contact and the line contact occur alternately. When the length of the contacted line accumulates enough to generate a new wave, a jump will occur to a higher order point contact state.

3) In the line contact state, the lengths of the contact lines may distribute arbitrarily in longitude. However, if they are concentrated to one segment, the new wave will be generated most easily.

4) As the axial force increasing, the point contact will occur while the axial force satisfies the condition $P / P_{\mathrm{E}} \in\left((2 n-1)^{2},(2 n)^{2}\right)$. In this situation, the free segment maintains its equilibrium by changing its deflection curve. On the other hand, the line contact will occur as $P / P_{\mathrm{E}} \in\left((2 n)^{2},(2 n+1)^{2}\right)$, during which the equilibrium is guaranteed by decreasing the length of the free segment and increasing the length of the contact lines. 
5) The contact force is proportional to the clearance between the core and the restraining component. It is also influenced by the axial force it sustained and the bending stiffness of the core plate.

\section{ACKNOWLEDGEMENT}

The authors would like to acknowledge financial supports from the National Natural Science Foundation (50878055, 51008077) and the Priority Academic Program Development of Jiangsu Higher Education Institutions.

\section{REFERENCES}

[1] Uang, C.M., Nakashima, M. and Tsai, K.C., "Research and Application of Buckling-Restrained Braced Frames”, International Journal of Steel Structures, 2004, Vol. 4, No. 4, pp. 301-313.

[2] Lai, J.W. and Tsai, K.C., "Research and Application of Buckling Restrained Braces in Taiwan”, ANCER Annual Meeting, Hawaii, USA, 2004.

[3] Xie, Q., "State of the Art of Buckling-Restrained Braces in Asia”, Journal of Constructional Steel Research, 2005, Vol. 61, No. 6, pp. 727-748.

[4] Luo, X.Q., Ge, H.B. and Usami, T., "Dynamic Numerical Simulation of Steel Frame-Typed Piers Installed with SMA Damping Devices Based on Multi-linear One Dimensional Constitutive Model”, International Journal of Advanced Steel Construction, 2010, Vol. 6, No. 2, pp. 722-741.

[5] Chen, Z.Y., Ge, H.B. and Usami, T., "Analysis and Design of Steel Bridge Structures with Energy Absorption Members”, International Journal of Advanced Steel Construction, 2008, Vol. 4, No. 3, pp. 173-183.

[6] Watanabe, A., Hitomi, Y., Saeki, E., et al., "Properties of Brace Encased in Buckling-restraining Concrete and Steel Tube”, Proceedings of $9^{\text {th }}$ World Conference on Earthquake Engineering, Tokyo-Kyoto, Japan, 1988, Vol. 4, pp. 719-724.

[7] Guo, Y.T. and Ren, W.M., "Some Advances in Confined Buckling”, Advances in Mechanics, 2004, Vol. 34, No. 1, pp. 41-52 (in Chinese).

[8] Sridhara, B.N., “Sleeved Compression Member”, USA: 5175972, 1993.

[9] Prasad, B.K., "Experimental Investigation of Sleeved Column”, Proceedings of the $33^{\text {rd }}$ AIAA/ASCE Structures, Structural Dynamics and Materials Conference, Dallas, USA, 1992, pp. 991-999.

[10] Domokos, G., Holmes, P. and Royce, B., “Constrained Euler Buckling”, Journal of Nonlinear Science, 1997, Vol. 7, No. 3, pp. 281-314.

[11] Ha, M.Q., "Study on the Mechanical Performances and Applications of Conventional and Innovative Buckling Restrained Braces”, Tongji University, 2004 (in Chinese).

[12] Chai, H., "The Post-bucking Response of a Bi-laterally Constrained Column“, Journal of the Mechanics and Physics of Solids, 1998, Vol. 46, No. 7, pp. 1151-1181.

[13] Shen, B. and Deng, C.G., "Continuous Transition from Point Contact to Line Contact between the Axially Compressed Inner Core and the Flexible Sleeve in a Sleeved Column”, Engineering Mechanics，2007, Vol. 24, No. 2, pp. 154-160 (in Chinese).

[14] Shen, B. and Deng, C.G., "Buckling Analysis of an Axially Compressed Strut Constrained by a Flexible Sleeve”, Mechanics in Engineering, 2006, Vol. 28, No. 5, pp. $43-46$ (in Chinese).

[15] Usami, T., Lu, Z.H. and Ge, H.B., "A Seismic Upgrading Method for Steel Arch Bridges Using Buckling-restrained Braces”, Earthquake Engineering and Structural Dynamics, 2005, Vol. 34, No. 4-5, pp. 471-496. 